\title{
A New High-Performance Liquid Chromatographic Method for the Determination and Distribution of Linalool in Michelia alba
}

\author{
En-Qin Xia ${ }^{1}$, Yang Song ${ }^{1}$, Xu-Xia Ai ${ }^{1}$, Ya-Jun Guo ${ }^{1}$, Xiang-Rong $\mathrm{Xu}^{2}$ and Hua-Bin $\mathrm{Li}^{{ }^{1} \text {, }}$
}

1 Guangdong Provincial Key Laboratory of Food, Nutrition and Health, School of Public Health, Sun Yat-Sen University, Guangzhou 510080, China; E-Mails: enqinxia@163.com (E.X.); sssongyang@163.com (Y.S.); 20040995axx@163.com (X.A.); guoyajunleo@163.com (Y.G.)

2 LMB, South China Sea Institute of Oceanology, Chinese Academy of Sciences, Guangzhou 510301, China; E-Mail: xuxr2000@yahoo.com (X.X.)

* Author to whom correspondence should be addressed; E-Mail: lihuabin@mail.sysu.edu.cn; Tel.: +86-20-8733-2391; Fax: +86-20-8733-0446.

Received: 23 June 2010; in revised form: 7 July 2010 / Accepted: 8 July 2010 /

Published: 12 July 2010

\begin{abstract}
A new high-performance liquid chromatographic method with photodiode array detection was established for the determination of linalool in the plant Michelia alba. Linalool was extracted from the plant sample with the aid of ultrasound, and was analyzed on a Waters RP $\mathrm{C}_{18}$ column $(4.6 \times 150 \mathrm{~mm}, 5 \mu \mathrm{m})$ using an acetonitrile and water $(55: 45$, $\mathrm{v} / \mathrm{v}$ ) mobile phase at a flow rate of $1.0 \mathrm{~mL} / \mathrm{min}$. The column temperature was set at $25^{\circ} \mathrm{C}$, and the detection wavelength was $210 \mathrm{~nm}$. The linear range of the method was $5-200 \mu \mathrm{g} / \mathrm{mL}$ with a correlation coefficient of 0.9975 . The recovery was $92-112 \%$, and the relative standard deviation was $1.85 \%(\mathrm{n}=9)$. The present method has been used to study the distribution of linalool in the plant Michelia alba. The plant samples include flowers, leaves and tender twigs. Furthermore, leaves included samples in their tender, grown-up and fallen phases, and flowers included samples in their juvenile, middle and whitening phases. The concentrations of linalool in different parts of the plant were $0.21-0.65 \%$, $1.63-4.89 \%$ and $0.43 \%$ for leaves, flowers and tender twigs, respectively. The results showed that all the plant materials contained relative high concentration of linalool, and juvenile phase flowers contained the highest concentration of linalool. Notably, the fallen leaves also contained high concentrations of linalool, which could be a potential resource of this compound. The results obtained are very helpful for the potential full utilization of this plant.
\end{abstract}


Keywords: linalool; distribution; high-performance liquid chromatography; Michelia alba

\section{Introduction}

Linalool (3,7-dimethyl-1,6-octadien-3-ol) is a monoterpene alcohol, which occurs naturally in several aromatic plants [1,2]. Linalool is a much sought after compound in the flavor and fragrance industry, and contributes to the characteristic aroma of a vast number of natural products, such as fruits and spices, as well as tea and chocolate $[3,4]$. Recent studies showed that linalool has some healthbenefits, such as anticancer, anti-inflammatory, antinociceptive, antimicrobial and antioxidant activities as well as mosquito- and sand fly-repelling action [5-8]. Therefore, the development of a simple and effective method for the determination of linalool in plant samples which could be used to screen or find new natural sources of linalool, and monitor the isolation and purification process of linalool from plant materials is of great importance.

In the literature, gas chromatography (GC) has been widely used for the determination of organic compounds [9-13], and has also been applied for the determination of linalool in plant samples [14-16]. Linalool in plant samples was usually determined by GC-MS [14-16], but linalool in extraction solutions that contain an organic solvent and water must be displaced to an organic solvent before injection onto a GC column, and this step could be omitted if the sample were determined by high-performance liquid chromatography (HPLC). In addition, GC-MS was not so widely used as HPLC-UV because GC-MS instruments are much more expensive than HPLC-UV instruments. Furthermore, the determination of the same sample by HPLC-UV often costs less money than for GCMS. On the other hand, although HPLC has been widely applied for the determination of many substances [17-26], no report about the determination of linalool in plant samples by HPLC could be found in the literature. Therefore, the aim of this study was to develop a simple, fast and efficient HPLC method for the determination of linalool in the plant Michelia alba that is widely planted in South China, and to further investigate the distribution of linalool in different parts of this plant. The results obtained should be useful for the full utilization of this species.

\section{Results and Discussion}

\subsection{Optimization of chromatographic condition}

According to the literature [18-20], a $\mathrm{C}_{18}$ column is often used in reversed-phase HPLC. In this study, a RP $\mathrm{C}_{18}$ column $(250 \mathrm{~mm} \times 4.6 \mathrm{~mm}, 5 \mu \mathrm{m})$ was tested with different mobile phases. Linalool is an oily compound, and would remain as a neutral molecule during the separation. Thus, the mobile phase may be a simple combination of organic solvent and water, and a buffer solution will not be needed. Methanol and acetonitrile are the most widely used mobile phase components in HPLC separation. However, it was very difficult to separate linalool from other components in the sample when different ratios of methanol-water were used as the mobile phases. In addition, the absorption of methanol was very strong at the detection wavelength of $210 \mathrm{~nm}$. Thus, acetonitrile and deionized water at different ratios were tried as the mobile phases for the separation of linalool from other 
components in the sample. The separation efficiency was investigated with the acetonitrile composition varying from $50-80 \%$ in the mobile phase. The results showed that linalool could be completely separated from other components in the sample with acetonitrile-water $(55: 45, \mathrm{v} / \mathrm{v})$ as a mobile phase, and the chromatographic peak of linalool was unattached and symmetrical with a retention time of about $8.5 \mathrm{~min}$. Therefore, acetonitrile-water $(55: 45, \mathrm{v} / \mathrm{v})$ was chosen as the mobile phase in the subsequent experiments. In addition, parameters of the detector were set at 3D mode, and the wavelength was scanned from $190 \mathrm{~nm}$ to $700 \mathrm{~nm}$ in order to select the optimum detection wavelength. The results showed that the best detection wavelength was $210 \mathrm{~nm}$. Thus, peak area of linalool at $210 \mathrm{~nm}$ was used to quantify linalool in the samples throughout this study. Representative chromatograms of a linalool standard and the extracts of Michelia alba are shown in Figure 1.

Figure 1. Chromatograms of standard linalool and the extracts of Michelia alba: (a) standard linalool, (b) extract of white flower, and (c) extract of tender leaves.

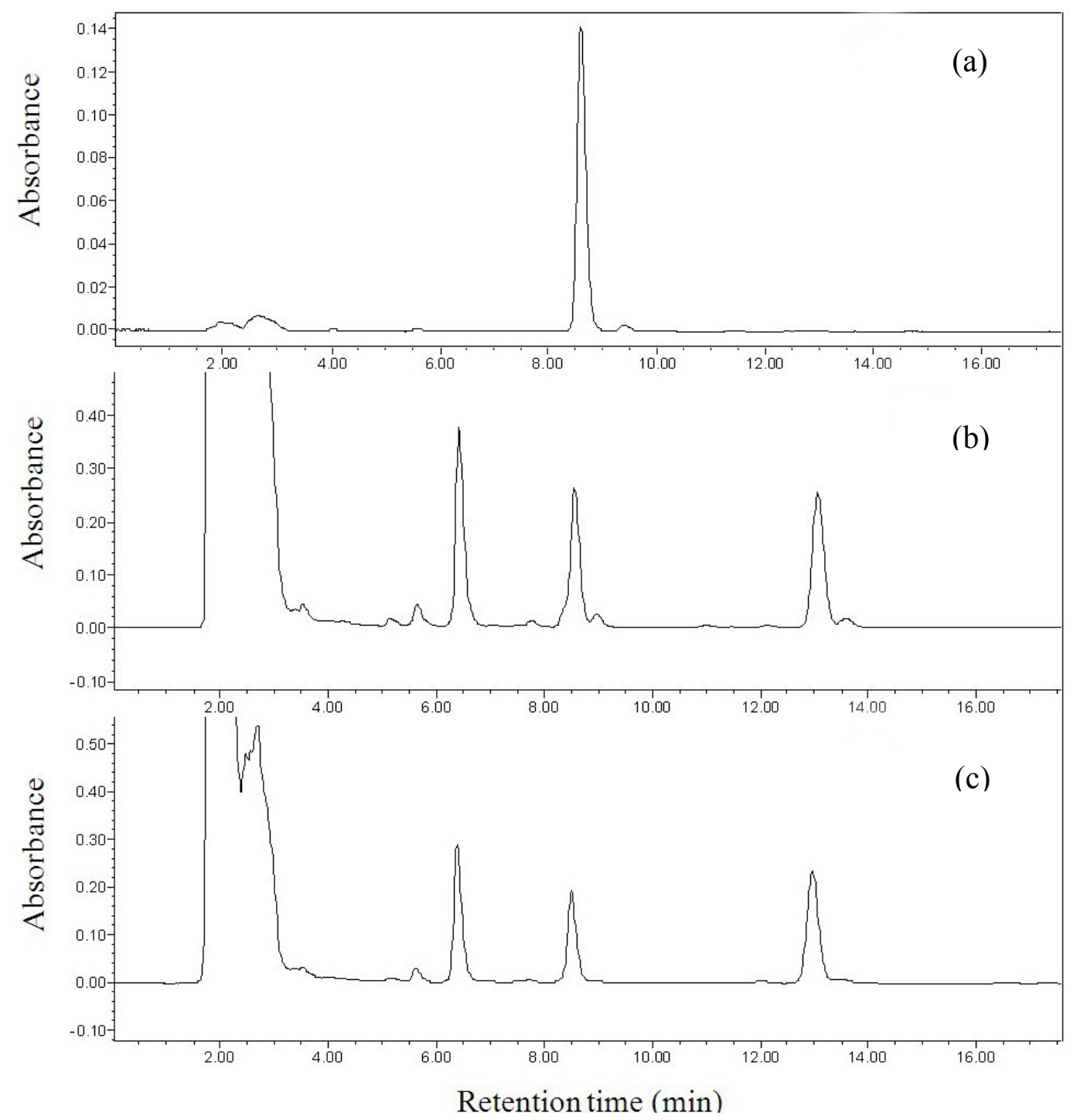




\subsection{Method validation}

The linearity of calibration curve was checked by preparing standard solutions of linalool at seven different concentrations using the stock solution. Each solution was injected in triplicate, and the mean peak area was used. The method showed a good linearity in concentrations ranging from 5 to $200 \mu \mathrm{g} / \mathrm{mL}$ with a correlation coefficient of 0.9975 , and the equation is $A$ (peak area) $=9296.8 C$ (concentration, $\mu \mathrm{g} / \mathrm{mL}$ ) - 29250. The limit of detection was $2 \mu \mathrm{g} / \mathrm{mL}$ based on a signal $/$ noise of 3:1 [27]. The precision of method was studied by repeatedly injecting the samples from whitening buds $(\mathrm{n}=9)$, and the relative standard deviation was $1.85 \%$. The recovery of the method was verified using samples with high and low concentrations in triplicate. The recoveries ranged from $92 \%$ to $112 \%$, and the relative standard deviations for high and low concentrations were $1.5 \%$ and $2.1 \%$, respectively.

\subsection{Distribution of linalool in the plant Michelia alba}

The present method has been used to study the distribution of linalool in the plant Michelia alba. Linalool was extracted from the plant samples with ethanol under ultrasonic irradiation, and then analyzed by the proposed method. The samples included different parts of plants, such as flowers, leaves and tender twigs. Furthermore, leaves include different samples in their tender, grown and fallen phases, and flowers in their juvenile, middle and whitening phases were also included. The results are presented in Table 1. From Table 1, the contents of the linalool for tender twigs, leaves and budding flowers were $0.44 \%, 0.21-0.65 \%, 1.63-4.89 \%$, respectively. It is apparent from the data that there is a rather large leap between the contents of linalool in leaves and flowers, and levels in twigs and leaves are similar. The content of linalool in juvenile flower buds was near ten times as high as that in leaves and twigs. The results did not agree with those of Mishra et al. [28], who reported quite similar levels of concentration of linalool in essential oil of leaves and flowers. The difference might come from different plants studied in each case: Lippia alba and Michelia alba. It is also possible that the samples in their study might be from blossomed flowers, which had released a lot of aromatic compounds before sampling.

Table 1. The content of linalool in the different parts of plant Michelia alba.

\begin{tabular}{cc}
\hline Parts of the plant & Content of linalool (\%, wet weight) \\
\hline tender leaves & $0.65 \pm 0.012$ \\
grown green leaves & $0.21 \pm 0.004$ \\
fallen leaves & $0.46 \pm 0.009$ \\
juvenile buds of flowers & $4.89 \pm 0.073$ \\
middle buds of flowers & $2.86 \pm 0.049$ \\
whitening buds of flowers & $1.63 \pm 0.033$ \\
tender twig & $0.44 \pm 0.011$ \\
\hline
\end{tabular}

Among different phases of budding flowers, juvenile buds contained the highest concentration of linalool, accounting for $4.89 \%$ in fresh samples. With the growth of buds, the concentration of linalool decreased gradually, and whitening buds contained the lowest levels of linalool. This phenomenon can be explained by two aspects. On the one hand, the aromatic compounds could be all present in the 
flowers when the buds came into being, and then the aromatic compound levels decreased during the growth of buds. On the other hand, the whitening buds indeed displayed a noticeable linalool aroma.

The content of linalool showed abnormal changes in the different phases of leaves (Table 1). The decreasing order was tender, fallen and growth green leaves, and their concentrations were $0.65 \%$, $0.46 \%$, and $0.21 \%$, respectively. The tender leaves contained the highest concentration of aromatic compounds. The content of linalool decreased gradually with the growth of leaves. The fallen leaves had less weight than the grown leaves due to the volatilization of the water, which resulted in an increased relative content of linalool in fallen leaves.

It was very important to find that the leaves contain quite high concentrations of linalool (Table 1), which were at the same level as those of the plant Lippia alba [1,2,4] (Table 2). Compared with the flowers, leaves constitute very a large part of the total plant, and they are conveniently available without injuring the living tree. Furthermore, the fallen leaves contain the relative high content of linalool, which indicated that fallen leaves are a potential resource of linalool.

Table 2. The contents of linalool (\%, weight) in the plant Lippia alba $[1,2,4]$

\begin{tabular}{lcc}
\hline Parts of the plant & Contents of linalool in plant & References \\
\hline Leaf of Lippia alba & $0.13 \%$ & {$[2]$} \\
Leaf of Lippia alba & $0.39-0.52 \%$ & {$[1]$} \\
Leaf of Lippia alba & $0.3-0.8 \%$ & {$[4]$} \\
\hline
\end{tabular}

\section{Experimental}

\subsection{Apparatus}

The HPLC system used throughout this study was a Waters (Milford, MA, USA) 1525 Binary HPLC Pump separations module, equipped with a Waters 2996 photodiode array detector. The system also included an auto-injector, and a Waters reversed-phase $C_{18}$ analytical column $(4.6 \mathrm{~mm} \times 250 \mathrm{~mm}$, $5 \mu \mathrm{m})$. The mobile phase was acetonitrile-water $(55: 45, \mathrm{v} / \mathrm{v})$ with a flow-rate of $1 \mathrm{~mL} / \mathrm{min}$, and the wavelength of detection was set at $210 \mathrm{~nm}$. The mobile phase was degassed with ultrasonic waves before operation. Evaluation and quantification were performed with an Empower chromatography system (Waters). The ultrasound-assisted extraction was carried out in a KQ-600E ultrasonic device (Changzhou Nuoji Instrument Company, China) with an ultrasound power of $600 \mathrm{~W}$, heating power of $800 \mathrm{~W}$, and frequencies of $40 \mathrm{kHz}$, equipped with digital timer and temperature controller.

\subsection{Reagents}

Linalool was bought from Sigma (St. Louis, MO, USA). Acetonitrile, methanol and ethanol were HPLC grade and were purchased from Merck (Germany). Deionized water was used throughout the experiments. A stock solution of linalool $(1,000 \mu \mathrm{g} / \mathrm{mL})$ was prepared in methanol and was stored at $4{ }^{\circ} \mathrm{C}$. The calibration standard solutions $(5-200 \mu \mathrm{g} / \mathrm{mL})$ were prepared from the stock solution by serial dilutions with methanol. 


\subsection{Plant Materials and samples treatment}

The leaves, flowers and tender twig of Michelia alba were collected in December 2009 from Guangzhou, Guangdong Province, China. The leaves and flowers were ground into small pieces and mixed with an appropriate amount of ethanol. After $30 \mathrm{~min}$, these suspensions were irradiated with ultrasound for $20 \mathrm{~min}$. All samples were filtered through $0.45-\mu \mathrm{m}$ nylon filters, and then injected onto the HPLC column. The samples of flowers were diluted to three times the initial volume with ethanol before injection. All the experiments were carried out in triplicate, and the results were expressed as mean or mean \pm SD (standard deviation).

\section{Conclusions}

A new high-performance liquid chromatographic method with photodiode array detection was established for the determination of linalool. The linear range of method was $5-200 \mu \mathrm{g} / \mathrm{mL}$ with a correlation coefficient of 0.9975 . The recovery was $92-112 \%$, and the relative standard deviation was $1.85 \%(\mathrm{n}=9)$. The present method was a simple, fast and efficient method for the determination of linalool in plant samples, and has been used to study the distribution of linalool in the plant Michelia alba. The concentrations of linalool in different parts of the plant were $0.21-0.65 \%, 1.63-4.89 \%$ and $0.43 \%$ for leaves, flowers and tender twigs, respectively. The results showed that all plant materials contained relative high concentration of linalool, among which juvenile phase flowers contained the highest concentration of linalool. Compared with the flowers, leaves constitute very a large part of the plant mass, and they could be used without injuring the living tree. Furthermore, the fallen leaves contain a relatively high content of linalool, which indicated that fallen leaves are a potential resource of linalool. The results obtained should be very helpful for the full utilization of this plant.

\section{Acknowledgements}

This research was supported by the Hundred-Talents Scheme of Sun Yat-Sen University. The technical assistance from Ling Zheng is highly appreciated.

\section{References}

1. Bahl, J.R.; Sinha, S.; Naqvi, A.A.; Bansal, R.P.; Gupta, A.K.; Kumar, S. Linalool-rich essential oil quality variants obtained from irradiated stem nodes in Lippia alba. Flavour Fragr. J. 2002, 17, 127-132.

2. Bahl, J.R.; Garg, S.N.; Singh, S.C.; Bansal, R.P.; Naqvi, A.A.; Kumar, S. Composition of linalool rich essential oil from Lippia alba grown in Indian plains. Flavour Fragr. J. 2000, 15, 199-200.

3. Bazemore, R; Rouseff, R; Naim, M. Linalool in orange juice: Origin and thermal stability. $J$. Agric. Food Chem. 2003, 51, 196-199.

4. Bonnländer, B., Cappuccio, R.; Liverani, F.S.; Winterhalter, P. Analysis of enantiomeric linalool ratio in green and roasted coffee. Flavour Fragr. J. 2006, 21, 637-641.

5. Kamatou, G.P.P.; Viljoen, A.M. Linalool - a review of a biologically active compound of commercial importance. Nat. Prod. Commun. 2008, 3, 1183-1192. 
6. Peana, A.T.; D'Aquila, P.S.; Panin, F.; Serra, G.; Pippia, P.; Moretti, M.D.L. Anti-inflammatory activity of linalool and linalyl acetate constituents of essential oils. Phytomedicine 2002, 9, 721-726.

7. Queiroga, C.L.; Teixeira D.M.C.; Ribeiro, B.B.; de Magalhaes, P.M. Linalool production from the leaves of Bursera aloexylon and its antimicrobial activity. Fitoterapia 2007, 78, 327-328.

8. Mueller, G.C.; Junnila, A.; Kravchenko, V.D.; Revay, E.E.; Butler, J.; Schlein, Y. Indoor protection against mosquito and sand fly bites: A comparison between citronella, linalool, and geraniol candles. J. Amer. Mosquito Contr. Assn. 2008, 24, 150-153.

9. Li, J.; Liu, X.G.; Dong, F.S.; Xu, J.; Zheng, Y.Q.; Shan, W.L. Determination of the volatile composition in essential oil of Descurainia sophia (L.) Webb ex Prantl (Flixweed) by gas chromatography/mass spectrometry (GC/MS). Molecules 2010, 15, 233-240.

10. Tang, Y.P.; Zhu, M.; Yu, S.; Hua, Y.Q.; Duan, J.A.; Su, S.L.; Zhang, X.; Lu, Y.; Ding, A.W. Identification and comparative quantification of bioactive phthalides in essential oils from Si-WuTang, Fo-Shou-San, Radix Angelica and Rhizoma Chuanxiong. Molecules 2010, 15, 341-351.

11. Kasiotis, K.M.; Souki, H.; Tsakirakis, A.N.; Carageorgiou, H.; Theotokatos, S.A.; Haroutounian, S.A.; Machera, K. Headspace solid-phase microextraction gas chromatographic determination of fenthion in human serum. Int. J. Mol. Sci. 2008, 9, 906-913.

12. Cigić, I.K.; Prosen, H. An overview of conventional and emerging analytical methods for the determination of mycotoxins. Int. J. Mol. Sci. 2009, 10, 62-115.

13. Li, L.; Zhao, J.C. Determination of the volatile composition of Rhodobryum giganteum (Schwaegr.) Par. (Bryaceae) using solid-phase microextraction and gas chromatography/mass spectrometry (GC/MS). Molecules 2009, 14, 2195-2201.

14. Shang, C.Q.; Hu, Y.M.; Deng, C.H.; Hu, K.J. Rapid determination of volatile constituents of Michelia alba flowers by gas chromatography-mass spectrometry with solid-phase microextraction. J. Chromatogr. A 2002, 942, 283-288.

15. Qin, C.G.; Lu, Z.E.; Chen, K.Q. Study on chemical constituents of essential oil of Michelia alba leaves by gas chromatography-mass spectrometry. Chin. J. Chromatogr. 1999, 17, 40-42.

16. Wang, X.Y.; Liu, M.C.; Yang, Y.W.; Deng, W.; Li, Z.G. Chemical constituents of the essential oil of Michelia alba DC by GC-MS. J. Chongqing Univ. 2008, 31, 97-100.

17. Xie, J.W.; Huang, L.F.; Hu, W.; He, Y.B.; Wong, K.P. Analysis of the main nucleosides in Cordyceps Sinensis by LC/ESI-MS. Molecules 2010, 15, 305-314.

18. Lu, H.T.; Li, H.B.; Chen, F.; Wong, M.H.; Jiang, Y. Determination of triphenyltin and its metabolite diphenyltin in culture medium by high-performance liquid chromatography with UV detection. Chromatographia 2004, 60, 113-116.

19. Li, H.B.; Chen, F. Simultaneous determination of nine water-soluble vitamins in pharmaceutical preparations by high-performance liquid chromatography with diode array detection. J. Sep. Sci. 2001, 24, 271-274.

20. Xu, X.R.; Li, H.B.; Gu, J.D.; Paeng, K.J. Determination of fluoride in water by reversed-phase high-performance liquid chromatography using $\mathrm{F}^{-}-\mathrm{La}^{3+}$-alizarin complexone ternary complex. Chromatographia 2004, 59, 745-747.

21. Oliveira, C.A.F.; Gonçalves, N.B.; Rosim, R.E.; Fernandes, A.M. Determination of aflatoxins in peanut products in the northeast region of São Paulo, Brazil. Int. J. Mol. Sci. 2009, 10, 174-183. 
22. Li, H.B.; Chen, F.; Xu X.R. Determination of iodide in seawater and urine by size exclusion chromatography with iodine-starch complex. J. Chromatogr. A 2001, 918, 335-339.

23. He, H.Z.; Li, H.B.; Chen, F. Determination of vitamin $B_{1}$ in seawater and microalgal fermentation media by high-performance liquid chromatography with fluorescence detection. Anal. Bioanal. Chem. 2005, 383, 875-879.

24. Li, H.B.; Chen, F. Simultaneous determination of twelve water- and fat- soluble vitamins by highperformance liquid chromatography with diode array detection. Chromatographia 2001, 54, 271-273.

25. Xu, X.R.; Li, H.B.; Gu, J.D.; Paeng, KJ. Determination of iodate in iodized salt by reversed-phase high-performance liquid chromatography with UV detection. Chromatographia 2004, 60, 721-723.

26. Li, J.; Xie, H.H.; Yang, B.; Dong, X.H.; Feng, L.Y.; Chen, F.; Jiang, Y.M. A comparative identification of ochratoxin $\mathrm{A}$ in longan fruit pulp by high-performance liquid chromatographyfluorescence detection and electron spray ionization-mass spectrometry. Molecules 2010, 15, 680-688.

27. Li, H.B.; Chen, F. Determination of silicate in water by ion exclusion chromatography with conductivity detection. J. Chromatogr. A 2000, 874, 143-147.

28. Mishra, R.K.; Chaudhary, S.; Pandey, R.; Gupta, S.; Mallavarapu, G.R.; Kumar, S. Analysis of linalool content in the inflorescence (flower) essential oil and leaf oil of Lippia alba cultivar 'Kavach'. J. Essent. Oil Res. 2010, 22, 3-7.

Sample Availability: The plant samples are available from the authors.

(C) 2010 by the authors; licensee MDPI, Basel, Switzerland. This article is an Open Access article distributed under the terms and conditions of the Creative Commons Attribution license (http://creativecommons.org/licenses/by/3.0/). 